\title{
Linearization and compactness
}

\author{
by \\ Jesús Ángel Jaramillo (Madrid), Ángeles Prieto (Madrid) and \\ Ignacio Zalduendo (Buenos Aires)
}

\begin{abstract}
This paper is devoted to several questions concerning linearizations of function spaces. We first consider the relation between linearizations of a given space when it is viewed as a function space over different domains. Then we study the problem of characterizing when a Banach function space admits a Banach linearization in a natural way. Finally, we consider the relevance of compactness properties in linearizations, more precisely, the relation between different compactness properties of a mapping, and compactness of its associated linear operator.
\end{abstract}

Introduction. Let $\mathcal{F}(U)$ be a linear space of continuous complex-valued functions on a topological space $U$. By a linearization of $\mathcal{F}(U)$ over $U$ we understand a pair $(Z, e)$, where $Z$ is a locally convex vector space and $e: U \rightarrow Z$ is a continuous map satisfying

(i) For every continuous linear functional $L \in Z^{\prime}$ we have $L \circ e \in \mathcal{F}(U)$.

(ii) For each $f \in \mathcal{F}(U)$ there exists a unique continuous linear functional $L_{f} \in Z^{\prime}$ such that $f=L_{f} \circ e$, that is, the following diagram commutes:

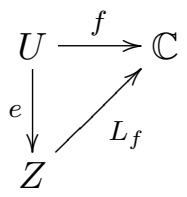

In this way, $\mathcal{F}(U)$ is identified algebraically with the dual space of $Z$. Linearization can be a useful tool for the study of function spaces, since it enables the application of linear functional analysis to problems concerning non-linear functions.

Tensor products are a typical example of such an object, but many other linearizations have been constructed for various kinds of function spaces. For example, when $\mathcal{F}(U)$ is the space of Lipschitz functions on a metric space $U$,

2000 Mathematics Subject Classification: 46E10, 46E50, 47B07.

Key words and phrases: linearizations, Banach linearization, factorization of compact mappings. 
the classical Arens-Eells construction provides a linearization of $\mathcal{F}(U)$ (see [23, Theorem 2.2.4]). For the space of continuous homogeneous polynomials on a Banach space, a linearization has been given by Ryan [21]. In the holomorphic setting, linearizations have been constructed by Mazet [15] and by Mujica and Nachbin [18] for spaces of holomorphic functions on finite- or infinite-dimensional domains. The case of bounded holomorphic functions was considered by Mujica [16], and the space of holomorphic functions of bounded type was studied by Galindo, García and Maestre [11] and by Mujica [17] (see also [3]). Some related work can be seen in [2]. Linearizations of several spaces of holomorphic functions have also been considered in [4], [5], and [12], where various properties of the corresponding linearizations are studied in connection with properties of the underlying spaces.

In [6] Carando and Zalduendo develop a general linearization procedure by constructing a canonical linearization $\left(\mathcal{F}_{*}(U), e\right)$ for any linear space of continuous functions $\mathcal{F}(U)$, which encompasses the above mentioned examples. This linearization also produces a factorization for vector-valued mappings in the following way. If $F$ is a locally convex space we denote by $\omega \mathcal{F}(U, F)$ the space of all continuous mappings $f: U \rightarrow F$ such that $\varphi \circ f \in \mathcal{F}(U)$ for every continuous linear functional $\varphi \in F^{\prime}$. It is proved in [6, Theorem 3] that for each $f \in \omega \mathcal{F}(U, F)$ there exists a continuous linear operator $L_{f}: \mathcal{F}(U) \rightarrow F$ such that the following diagram commutes:

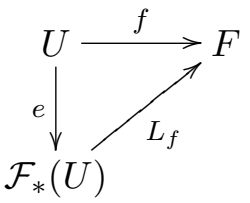

In this way, we can identify algebraically $\omega \mathcal{F}(U, F)$ with the space of continuous linear operators $\mathcal{L}\left(\mathcal{F}_{*}(U), F\right)$.

One of the purposes of this paper is to explore the relationship between compactness and linearizations, in particular, the relationship between different compactness properties of a mapping $f: U \rightarrow F$ and compactness (or weak compactness) of the corresponding operator $L_{f}: \mathcal{F}_{*}(U) \rightarrow F$. This problem has been studied by Pełczyński [20], Ryan [22], Aron and Schottenholer [1], and Mujica [16] for spaces of polynomials and holomorphic mappings in infinite dimensions. We provide here a general approach which extends and unifies some previous results.

For convenience, we begin by recalling the linearization procedure in [6]. We fix a topological space $U$ and a linear space $\mathcal{F}(U)$ of complex-valued continuous functions defined over $U$.

Consider first the vector space $\mathbb{C}^{(U)}$ of finitely supported families of $U$-indexed complex numbers. A typical element will be denoted by $s=$ $\sum_{x \in U} a_{x} e_{x}$, with $e_{x}(y)=\delta_{x y}$. Note that the sum is finite. For any given 
$f \in \mathcal{F}(U)$, define the seminorm

$$
p_{f}(s)=\left|\sum_{x \in U} a_{x} f(x)\right|,
$$

and

$$
\mathcal{N}=\left\{s \in \mathbb{C}^{(U)}: p_{f}(s)=0 \text { for all } f \in \mathcal{F}(U)\right\}
$$

Now define

$$
X=\mathbb{C}^{(U)} / \mathcal{N} .
$$

Continue to denote the class of $e_{x}$ by $e_{x}$, and the class of $s$ by $s=\sum_{x \in U} a_{x} e_{x}$. Note that now this way of writing the class of $s$ need not be unique. However, the seminorms on $X$, coming from $\left|\sum_{x \in U} a_{x} f(x)\right|$ (which we continue to denote $p_{f}$ ) are well-defined and provide a Hausdorff locally convex space structure for $X$. Also, define

$$
e: U \rightarrow X, \quad e(x)=e_{x} .
$$

It is clear that a function $f \in \mathcal{F}(U)$ factors through $e$ in the following way:

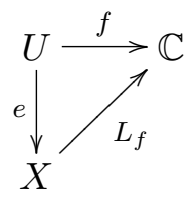

where $L_{f}$ is the continuous linear form defined by $L_{f}(s)=\sum_{x} a_{x} f(x)$ if $s=\sum_{x} a_{x} e_{x}$. The pairing $\langle X, \mathcal{F}(U)\rangle$ given by $\langle s, f\rangle=L_{f}(s)$ is a dual pairing, so algebraically we have $X^{\prime}=\mathcal{F}(U)$ (see [14]).

Consider now the topology $\tau$ on $X$ defined by means of the seminorms $p_{f}$, where $f$ ranges over all functions of $\mathcal{F}(U)$. There are of course many topologies on $X$ which are compatible with $\tau$ (i.e., topologies with the same continuous linear functionals). For any one of them the dual space $X^{\prime}$ is identified algebraically with $\mathcal{F}(U)$.

Define on $X$ the strongest locally convex topology compatible with $\tau$ for which the map $e: U \rightarrow X$ is continuous. We call it the $\alpha$-topology on $X$. Now $\mathcal{F}_{*}(U)$ is defined to be the completion of $(X, \alpha)$. This is the construction in [6]. Note that, as shown in [6], all the linearizations mentioned above are particular cases of this abstract linearization. We will use a few results from [6]. First, we have a characterization of the $\alpha$-topology:

FACT 0.1 ([6, Proposition 3]). The topology $\alpha$ on $\mathcal{F}_{*}(U)$ is the topology of uniform convergence on the equicontinuous pointwise compact disks of $\mathcal{F}(U)$.

The following uniqueness result will be also useful:

FACT 0.2 ([6, Corollary 2]). If $(Y, \widehat{e})$ is a linearization of $\mathcal{F}(U)$ and $Y$ is a Fréchet space, then there exists a topological isomorphism $T: \mathcal{F}_{*}(U) \rightarrow Y$ 
such that the following diagram commutes.

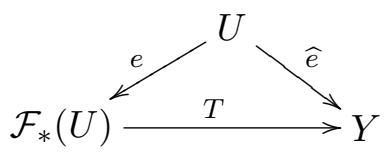

Now suppose that $\mathcal{F}(U)$ is endowed with a locally convex topology. A linearization $(Z, e)$ of $\mathcal{F}(U)$ is said to be strong if $\mathcal{F}(U)$ is topologically isomorphic to the strong dual $\left(Z^{\prime}, \beta\right)$. The next result gives a sufficient condition for $\mathcal{F}_{*}(U)$ to be strong.

Recall from [6] that a subset $B \subset X$ is called $\mathcal{F}$-bounded if it is bounded in the $\alpha$-topology (or any compatible topology), while a subset $A \subset U$ is called $\mathcal{F}$-bounding if every $f \in \mathcal{F}(U)$ is bounded on $A$. We say that $U$ has the $B B \mathcal{F}$-property if for each $\mathcal{F}$-bounded subset $B$ of $X$, there is an $\mathcal{F}$-bounding subset $A$ of $U$ and an $r>0$ such that

$$
B \subset r \cdot \operatorname{coe}(e(A)),
$$

where $\operatorname{coe}(e(A))$ denotes the closed absolutely convex hull of $e(A)$. Then we have

FACT 0.3 ([6, Theorem 1]). Suppose that $\mathcal{F}(U)$ is barreled, and its topology is that of uniform convergence on $\mathcal{F}$-bounding subsets of $U$. Then

(i) $\mathcal{F}(U)$ is isomorphic to the strong dual $\left(\mathcal{F}_{*}(U)^{\prime}, \beta\right)$,

(ii) $U$ has the $B B \mathcal{F}$-property.

The contents of the paper are as follows. Since many spaces can be modeled as function spaces in several different ways, we begin in Section 1 by studying the linearizations obtained when considering the same function space over several different domains. In Section 2 we consider the following problem: when $\mathcal{F}(U)$ is a Banach space of continuous funtions on $U$, a linearization is in general only a locally convex space. We are interested in giving conditions under which $\mathcal{F}(U)$ has a strong Banach linearization. Of course $\mathcal{F}(U)$ must be a dual Banach space, but we will see that this is not enough; a Banach space may have a Banach predual without having a linearizing Banach predual. We obtain a characterization in terms of compactness of the unit ball of $\mathcal{F}(U)$ for pointwise and compact-open topologies. This is akin to the Dixmier-Ng Theorem [19], but the result presented here is independent. In Section 3 we focus on the relationship between compactness properties of a mapping and compactness of its linearization. Apart from the usual definition of compactness, we also consider what we call boundedly compact mappings.

1. Function spaces over different domains. Many spaces can be viewed as spaces of continuous functions over several different domains, which can give rise to different linearizations. 
Example 1. Consider the Banach space $L^{1}[0,1]$. Any Banach space $E$ may be viewed as a space of continuous functions on $\left(B_{E^{\prime}}, \omega^{*}\right)$, by identifying each $x \in E$ with $\widehat{x}$ where $\widehat{x}(\gamma)=\gamma(x)$ for every $\gamma \in B_{E^{\prime}}$. Thus in particular

$$
L^{1}[0,1]=\mathcal{F}\left(B_{L^{\infty}}\right) .
$$

If $E$ is any Banach space with a Schauder basis $\left(v_{k}\right)_{k \in \mathbb{N}}$, we denote by $\left(v_{k}^{\prime}\right)_{k \in \mathbb{N}}$ the corresponding coordinate functionals, i.e. $x=\sum_{k=1}^{\infty} v_{k}^{\prime}(x) v_{k}$ for each $x \in E$. We may then consider $E$ as a space of (continuous) functions over $\mathbb{N}$ by identifying each element $x \in E$ with the sequence of its coordinates $\left(v_{1}^{\prime}(x), v_{2}^{\prime}(x), \ldots\right)$. In this way we see that

$$
L^{1}[0,1]=\mathcal{F}(\mathbb{N}) .
$$

Finally, if $A, B \in \mathcal{B}$ (the Borel $\sigma$-algebra of $[0,1]$ ), put $d(A, B)=$ $m(A \triangle B)$, and $A \sim B$ if $d(A, B)=0$. Then $d$ is a metric on $\mathcal{B} / \sim$, and each $f \in L^{1}[0,1]$ may be identified with the following map on $\mathcal{B} / \sim$, which is continuous:

$$
A \mapsto \int_{A} f d m
$$

In this way we find that

$$
L^{1}[0,1]=\mathcal{F}(\mathcal{B} / \sim) .
$$

The construction of the linearization $\mathcal{F}_{*}(U)$ corresponding to $\mathcal{F}(U)$ heavily depends on the domain $U$, and even on the topology of $U$, as the following simple example shows.

EXAmple 2. Let $E$ be a Banach space, and consider the two topological spaces $U=(E, w)$ and $V=(E,\|\|)$. The dual $E^{\prime}$ may then be viewed as

$E^{\prime}=\mathcal{F}(U)$ : the space of weakly continuous linear forms on $E$, or

$E^{\prime}=\mathcal{G}(V)$ : the space of norm continuous linear forms on $E$.

On linearizing, one obtains $\mathcal{F}_{*}(U)=U=(E, w)$ and $\mathcal{G}_{*}(V)=V=(E,\|\|)$.

We must, therefore, consider linearizations of function spaces over different domains, and ask ourselves when such linearizations coincide. We have the following result.

Proposition 1.1. Let $U$ and $V$ be topological spaces, $\mathcal{F}(U)$ and $\mathcal{G}(V)$ linear spaces of functions which are continuous over $U$ and $V$ respectively, and $\varphi: V \rightarrow U$ continuous and such that $f \circ \varphi \in \mathcal{G}(V)$ for each $f \in \mathcal{F}(U)$. Suppose that the transpose $\varphi^{t}: \mathcal{F}(U) \rightarrow \mathcal{G}(V)$ given by $\varphi^{t}(f)=f \circ \varphi$ is an algebraic isomorphism, and $\mathcal{F}_{*}(U)$ is a Fréchet space. Then there exists a topological isomorphism $T_{\varphi}: \mathcal{G}_{*}(V) \rightarrow \mathcal{F}_{*}(U)$ such that following diagram 
commutes:

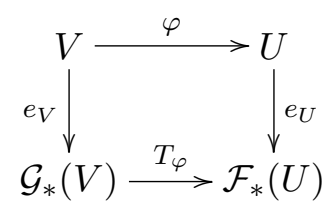

Proof. In what follows, we index with $U$ and $V$ objects appearing in the constructions of $\mathcal{F}(U)$ and $\mathcal{G}(V)$ respectively. Define $\widetilde{\varphi}: \mathbb{C}^{(V)} \rightarrow \mathbb{C}^{(U)}$ by $\tilde{\varphi}\left(\sum_{y \in V} a_{y} e_{y}\right)=\sum_{y \in V} a_{y} e_{\varphi(y)}$. It is easily seen that $\widetilde{\varphi}$ passes to the quotient, producing a map

$$
\bar{\varphi}: X_{V} \rightarrow X_{U}
$$

for which $L_{f} \circ \bar{\varphi}=L_{f \circ \varphi}$. Also, this map is continuous for the corresponding $\alpha$-topologies in $X_{V}$ and $X_{U}$. To see this, it will be enough to check that given any equicontinuous $U$-pointwise compact disk $A \subset \mathcal{F}(U)$, the set $\varphi^{t}(A)=\{f \circ \varphi: f \in A\}$ is an equicontinuous $V$-pointwise compact disk in $\mathcal{G}(V)$. It is clearly a disk, and pointwise compactness follows from the continuity (in the pointwise topologies) of $\varphi^{t}: \mathcal{F}(U) \rightarrow \mathcal{G}(V)$. To see the equicontinuity, fix $y \in V$ and $\varepsilon>0$. There is a neighborhood $W$ of $\varphi(y)$ such that for all $x \in W$ and $f \in A,|f(\varphi(y))-f(x)|<\varepsilon$. Thus for all $z$ in the neighborhood $\varphi^{-1}(W)$ of $y$, and for all $f \in A$,

$$
|f(\varphi(y))-f(\varphi(z))|<\varepsilon .
$$

Thus a map $\bar{\varphi}: \mathcal{G}_{*}(V) \rightarrow \mathcal{F}_{*}(U)$ is induced which is linear and continuous, and such that the transpose $\bar{\varphi}^{t}$ "coincides" with $\varphi^{t}$ in the sense that

$$
\bar{\varphi}^{t}\left(L_{f}\right)=L_{f} \circ \bar{\varphi}=L_{f \circ \varphi}=L_{\varphi^{t}(f)} .
$$

Now, since $\varphi^{t}: \mathcal{F}(U) \rightarrow \mathcal{G}(V)$ is an algebraic isomorphism, so is $\bar{\varphi}^{t}$ : $\mathcal{F}_{*}(U)^{\prime} \rightarrow \mathcal{G}_{*}(V)^{\prime}$. Then considering for each $f \in \mathcal{F}(U)$ the commutativity of the diagram

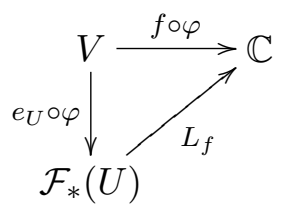

we see that $\left(\mathcal{F}_{*}(U), e_{U} \circ \varphi\right)$ is a linearization of $\mathcal{G}(V)$. Since $\mathcal{F}_{*}(U)$ is a Fréchet space, Fact 0.2 gives the desired isomorphism $T_{\varphi}: \mathcal{G}_{*}(V) \rightarrow \mathcal{F}_{*}(U)$.

A situation where Proposition 1.1 applies is the following. Let $U$ be an open connected subset of a locally convex space and $\mathcal{F}(U)$ a linear space of holomorphic functions on $U$ such that $\mathcal{F}_{*}(U)$ is a Fréchet space. If now $V \subset U$ is any non-empty open subset, let us consider the inclusion map $\iota: V \hookrightarrow U$ and the space

$$
\mathcal{G}(V)=\left\{\left.f\right|_{V}: f \in \mathcal{F}(U)\right\} .
$$


Since $U$ is connected, the restriction map $\mathcal{F}(U) \rightarrow \mathcal{G}(V)$ is an algebraic isomorphism and thus $\mathcal{G}_{*}(V)$ is topologically isomorphic to $\mathcal{F}_{*}(U)$ through the associated $T_{\iota}$ which makes the corresponding diagram commutative.

2. Banach linearization. Suppose that $(\mathcal{F}(U),\|\cdot\|)$ is a Banach space of continuous functions on a topological space $U$. In this section we consider the problem of when $\mathcal{F}(U)$ admits a strong Banach linearization $(Z, e)$. Recall that this means that $Z$ is isomorphic to a Banach space and the Banach dual $Z^{\prime}$ is isomorphic to $\mathcal{F}(U)$. In particular, we study when $\mathcal{F}_{*}(U)$ is a strong Banach linearization. Note that the topology of $\mathcal{F}(U)$ plays no role whatsoever in the construction of $\mathcal{F}_{*}(U)$, so in order to obtain our results relating the topologies of $\mathcal{F}_{*}(U)$ and $\mathcal{F}(U)$, we must necessarily impose some topological conditions on $\mathcal{F}(U)$.

For the case in which $U$ is a $k$-space, we obtain the following sufficient condition. Here, we denote by $\tau_{\text {co }}$ the compact-open topology and by $\tau_{\mathrm{p}}$ the pointwise topology on $\mathcal{F}(U)$.

TheOREM 2.1. Let $U$ be a $k$-space and $(\mathcal{F}(U),\|\cdot\|)$ a Banach space of continuous functions on $U$. If the ball of $\mathcal{F}(U)$ is $\tau_{\mathrm{co}}$-compact, then $\mathcal{F}_{*}(U)$ is a strong Banach linearization.

Proof. Let $B$ be the unit ball of $\mathcal{F}(U)$, which is $\tau_{\text {co-compact. For each }}$ $s \in X$, we may consider $s: \mathcal{F}(U) \rightarrow \mathbb{C}$ given by $s(f)=L_{f}(s)$. In this way, $s$ can be seen as a $\sigma(\mathcal{F}(U), X)$-continuous linear functional, so by compactness $|s|$ attains its maximum on $B$. Define

$$
\|s\|_{B}=\max _{B}|s| .
$$

This defines a norm on $X$. Note that for each $x \in U$, if $f \in \mathcal{F}(U)$ and $f \neq 0$, we have

$$
\left|e_{x}(f)\right|=\|f\| \cdot\left|e_{x}\left(\frac{f}{\|f\|}\right)\right| \leq\|f\| \cdot\left\|e_{x}\right\|_{B} .
$$

This shows that $e_{x} \in \mathcal{F}(U)^{\prime}$. We claim that $e: U \rightarrow\left(\mathcal{F}(U)^{\prime},\|\cdot\|\right)$ is continuous. Since $U$ is a $k$-space, it is enough to show that for each compact subset $K \subset U,\left.e\right|_{K}: K \rightarrow\left(\mathcal{F}(U)^{\prime},\|\cdot\|\right)$ is continuous. By the Ascoli-Arzelà Theorem, $\left.B\right|_{K}$ is equicontinuous on $K$. Hence, for every $\varepsilon>0$ and every $x \in K$, there exists a neighborhood $W^{x}$ such that if $y \in K \cap W^{x}$, then

$$
\sup \{|f(x)-f(y)|: f \in B\}<\varepsilon ;
$$

thus,

$$
\left\|e_{x}-e_{y}\right\|_{\mathcal{F}(U)^{\prime}}=\sup _{f \in B}\left|e_{x}(f)-e_{y}(f)\right|<\varepsilon .
$$

This establishes the claim. 
As a consequence, $B$ is in fact equicontinuous as a set of functions on $U$. Indeed, for $\varepsilon>0$ and every $x \in U$, there exists a neighborhood $V^{x}$ such that if $y \in V^{x}$, then

$$
\sup _{f \in B}|f(x)-f(y)|=\sup _{f \in B}\left|e_{x}(f)-e_{y}(f)\right|=\left\|e_{x}-e_{y}\right\|_{\mathcal{F}(U)^{\prime}}<\varepsilon .
$$

In this way we see that, since $B$ is an equicontinuous $\tau_{\mathrm{p}}$-compact disk, the norm $\|\cdot\|_{B}$ is continuous for the $\alpha$-topology. If we denote by $Y$ the completion of $\left(X,\|\cdot\|_{B}\right)$ we find that $e: U \rightarrow(X, \alpha) \rightarrow Y$ is continuous. Furthermore, each $f \in \mathcal{F}(U)$ linearizes through $Y$; indeed,

$$
\left|L_{f}(s)\right|=|s(f)|=\|f\|\left|s\left(\frac{f}{\|f\|}\right)\right| \leq\|f\| \cdot\|s\|_{B},
$$

so $L_{f}$ is $\|\cdot\|_{B}$-continuous. Now by Fact 0.2 we conclude that $\mathcal{F}_{*}(U)$ is isomorphic to the Banach space $Y$. Next, we are going to see that $\mathcal{F}_{*}(U)$ is strong. Note that $\mathcal{F}(U)$ can be algebraically identified with the dual space $Y^{\prime}$, and we can consider on $\mathcal{F}(U)$ the norm $\|\cdot\|_{d}$ which is dual to the norm of $Y$. It is clear that for every $x \in U$ and every $f \in \mathcal{F}(U)$,

$$
|f(x)| \leq\left\|e_{x}\right\|_{B} \cdot\|f\|_{d} .
$$

As a consequence, the topology induced by $\|\cdot\|_{d}$ on $\mathcal{F}(U)$ is finer than the $\tau_{\mathrm{p}}$-topology. On the other hand, as we have seen above, for every $x \in U$ and every $f \in \mathcal{F}(U)$,

$$
|f(x)| \leq\left\|e_{x}\right\|_{B} \cdot\|f\|,
$$

so the topology induced by $\|\cdot\|$ on $\mathcal{F}(U)$ is also finer than the $\tau_{\mathrm{p}}$-topology. Now, by applying the Closed Graph Theorem we find that, in fact, $\|\cdot\|_{d}$ is equivalent to $\|\cdot\|$ on $\mathcal{F}(U)$.

In the next theorem, by an equivalent ball in $\mathcal{F}(U)$ we mean the unit ball of a norm in $\mathcal{F}(U)$ which is equivalent to the original norm. We have to consider equivalent balls since, in general, a dual Banach space may have equivalent norms which are not dual norms (see, e.g., [10]).

TheOrem 2.2. Let $(\mathcal{F}(U),\|\cdot\|)$ be a Banach space of continuous functions on $U$. The following conditions are equivalent.

(i) $\mathcal{F}(U)$ admits a strong Banach linearization.

(ii) $\mathcal{F}_{*}(U)$ is a strong Banach linearization.

(iii) $\mathcal{F}(U)$ admits an equicontinuous and $\tau_{\mathrm{p}}$-compact equivalent ball.

(iv) $\mathcal{F}(U)$ admits an equivalent ball which is $\tau_{\mathrm{p}}$-compact, and the evaluation map $\delta: U \rightarrow\left(\mathcal{F}(U)^{\prime},\|\cdot\|\right)$ is continuous.

Furthermore, all of these conditions imply

(v) $\mathcal{F}(U)$ admits a $\tau_{\mathrm{co}}$-compact equivalent ball. 
Proof. (i) $\Leftrightarrow$ (ii) follows from Fact 0.2 .

(ii) $\Rightarrow$ (iii): Consider $\mathcal{F}(U)$ as the dual space of the Banach space $\mathcal{F}_{*}(U)$, endowed with the dual norm. The $w^{*}$-topology on $\mathcal{F}(U)$ is finer than $\sigma(\mathcal{F}(U), X)$, that is, the identity mapping

$$
\left(\mathcal{F}(U), w^{*}\right) \rightarrow(\mathcal{F}(U), \sigma(\mathcal{F}(U), X))
$$

is continuous. Note that the topology $\sigma(\mathcal{F}(U), X)$ coincides with $\tau_{\mathrm{p}}$ on $\mathcal{F}(U)$. Since the unit ball $B$ of $\mathcal{F}(U)$ is $w^{*}$-compact, it is $\tau_{\mathrm{p}}$-compact. On the other hand, $B$ is equicontinuous as a set of functions on $\mathcal{F}_{*}(U)$; since $e: U \rightarrow \mathcal{F}_{*}(U)$ is continuous, $B$ is an equicontinuous subset of $\mathcal{F}(U)$, considered as a set of functions on $U$.

(iii) $\Rightarrow$ (ii): We follow the lines of Theorem 2.1. Let $B$ be an equivalent ball in $\mathcal{F}(U)$ which is equicontinuous and $\tau_{\mathrm{p}}$-compact, and thus $\sigma(\mathcal{F}(U), X)$ compact. Each $s \in X$ can be viewed as a $\sigma(\mathcal{F}(U), X)$-continuous linear functional $s: \mathcal{F}(U) \rightarrow \mathbb{C}$, given by $s(f)=L_{f}(s)$. So $|s|$ attains its maximum on $B$. Define

$$
\|s\|_{B}=\max _{B}|s| .
$$

This is a norm on $X$ and we denote the corresponding completion of $X$ by $Y$. First, we check that $\mathcal{F}_{*}(U)$ is isomorphic to the Banach space $Y$. Note that the norm $\|\cdot\|_{B}$ is continuous for the $\alpha$-topology, since $B$ is an equicontinuous $\tau_{\mathrm{p}}$-compact disk. Therefore, the map $e: U \rightarrow Y$ is continuous. Also, each $f \in \mathcal{F}(U)$ linearizes through $Y$ : if $f \in \mathcal{F}(U)$, and $f \neq 0$, then for some $c>0$ we have $f / c\|f\| \in B$, and

$$
\left|L_{f}(s)\right|=|s(f)|=c \cdot\|f\| \cdot\left|s\left(\frac{f}{c\|f\|}\right)\right| \leq c \cdot\|f\| \cdot\|s\|_{B},
$$

so $L_{f}$ is $\|\cdot\|_{B}$-continuous. Now by Fact $0.2, \mathcal{F}_{*}(U)$ is isomorphic to $Y$. Finally, it can be proved as in Theorem 2.1 that $\mathcal{F}_{*}(U)$ is, in fact, a strong linearization.

(iii) $\Leftrightarrow$ (iv): Let $B$ be a $\tau_{\mathrm{p}}$-compact equivalent ball in $\mathcal{F}(U)$. Each evaluation functional $\delta_{x}: \mathcal{F}(U) \rightarrow \mathbb{C}$ is bounded on $B$ and therefore normcontinuous, where $\delta_{x}(f)=f(x)$ for each $x \in U$ and $f \in \mathcal{F}(U)$. It is clear that the evaluation map

$$
\delta: U \rightarrow\left(\mathcal{F}(U)^{\prime},\|\cdot\|\right)
$$

is continuous if and only if $B$ is an equicontinuous set of functions on $U$.

(iii) $\Rightarrow(\mathrm{v})$ : This is clear, since on an equicontinuous set, the topologies $\tau_{\mathrm{p}}$ and $\tau_{\text {co }}$ coincide.

Two direct consequences are the following.

COROLlary 2.3. If $U$ is a k-space, (i) through (v) in Theorem 2.2 above are all equivalent. 
COROLlary 2.4. Let $K$ be an infinite compact set, and $\mathcal{F}(K)$ an infinitedimensional closed subspace of $\left(C(K),\|\cdot\|_{\infty}\right)$. Then $\mathcal{F}(K)$ does not admit a Banach linearization.

Proof. Since $K$ is a compact set, $\tau_{\text {co }}=\|\cdot\|_{\infty}$ on $C(K)$. Now if $\mathcal{F}(K)$ admits a Banach linearization $(Z, e)$, then $\mathcal{F}(K)$ can be algebraically identified with the dual space $Z^{\prime}$, and we can consider on $\mathcal{F}(K)$ the norm $\|\cdot\|$ which is dual to the norm of $Z$. By Theorem 2.2, since $(Z, e)$ is a strong Banach linearization of $(\mathcal{F}(K),\|\cdot\|)$, this space admits an equivalent ball $B$ which is compact for $\tau_{\text {co }}=\|\cdot\|_{\infty}$. In particular, $B$ is $\|\cdot\|_{\infty}$-bounded and by the Open Mapping Theorem, $\|\cdot\|$ is equivalent to $\|\cdot\|_{\infty}$. The compactness of $B$ implies that $\mathcal{F}(K)$ is finite-dimensional.

Note, however, that when $K$ is hyperstonean, $C(K)$ is a dual Banach space by the Dixmier-Grothendieck Theorem [13]. Thus, admitting a strong Banach linearization is strictly stronger than admitting a Banach predual. A further example in this line is the following. We can consider any Banach space $E=\mathcal{F}\left(B_{E^{\prime}}\right)$ as a space of continuous functions on the dual unit ball $B_{E^{\prime}}$ with the $w^{*}$-topology, through the canonical inclusion map $E \hookrightarrow$ $C\left(B_{E^{\prime}}\right)$. Now, $E=\mathcal{F}\left(B_{E^{\prime}}\right)$ admits a Banach predual whenever $E$ is a dual space. Nevertheless, by Corollary $2.4, \mathcal{F}\left(B_{E^{\prime}}\right)$ admits a Banach linearization only when $E$ is finite-dimensional.

As mentioned before, many Banach spaces $E$ may be viewed as a space of functions $\mathcal{F}(U)$ in different ways. In particular, if $E$ is a Banach space with Schauder basis $\left(v_{k}\right)_{k \in \mathbb{N}}$ and we denote by $\left(v_{k}^{\prime}\right)_{k \in \mathbb{N}}$ the coordinate functionals, we may consider $E=\mathcal{F}(\mathbb{N})$ as a space of (continuous) functions over $\mathbb{N}$ by identifying each element $x \in E$ with the sequence of its coordinates $\left(v_{1}^{\prime}(x), v_{2}^{\prime}(x), \ldots\right)$. Note that, in this case, the evaluation map $\delta: \mathbb{N} \rightarrow\left(\mathcal{F}(\mathbb{N})^{\prime},\|\cdot\|\right)$ is well-defined and continuous.

We ask when $\mathcal{F}_{*}(\mathbb{N})$ is a strong Banach linearization. This is not always the case as, for example, when $\mathcal{F}(\mathbb{N})=c_{0}$. Indeed, if $B$ is a ball equivalent to the unit ball of $c_{0}$, then $B$ contains a sequence of the form $x_{n}=$ $(a, \stackrel{(n)}{.}, a, 0, \ldots)$ (where $a \neq 0$, and there are $n a$ 's, followed by 0 's). This sequence converges pointwise to $x=(a, a, a, \ldots)$, which is not an element of $c_{0}$, so $B$ is not pointwise compact. More generally, we have the following corollary. Here, (iii) $\Rightarrow$ (i) is essentially Alaoglu's Theorem (Theorem 6.10 in [10]).

Corollary 2.5. Let $E$ be a Banach space with a Schauder basis $\left(v_{k}\right)_{k \in \mathbb{N}}$, and consider $E=\mathcal{F}(\mathbb{N})$ as before. The following conditions are equivalent.

(i) $\mathcal{F}_{*}(\mathbb{N})$ is a strong Banach linearization.

(ii) Some equivalent ball of $E$ is $\tau_{\mathrm{p}}$-compact.

(iii) $\left(v_{k}\right)_{k \in \mathbb{N}}$ is boundedly complete. 
Proof. (i) $\Leftrightarrow$ (ii) follows from Theorem 2.2.

To prove (ii) $\Leftrightarrow$ (iii), suppose (ii) holds and let $\|\cdot\|$ be an equivalent norm on $E$ whose unit ball $B$ is $\tau_{\mathrm{p}}$-compact. Let $\left(z_{n}\right)$ be a sequence of complex numbers such that

$$
\sup _{n}\left\|\sum_{k=1}^{n} z_{k} v_{k}\right\|<\infty .
$$

We may - by normalizing - suppose this supremum to be 1 . The sequence $\left(x_{n}\right)$ given by $x_{n}=\sum_{k=1}^{n} z_{k} v_{k}$ is in $B$ and for each $k, v_{k}^{\prime}\left(x_{n}\right) \rightarrow z_{k}$ as $n \rightarrow \infty$ (indeed, the sequence is eventually $z_{k}$ ). By compactness, there is a subnet $x_{n_{i}}$ that converges pointwise to some $z \in B$. For each $k, v_{k}^{\prime}\left(x_{n_{i}}\right) \rightarrow v_{k}^{\prime}(z)$, so that $v_{k}^{\prime}(z)=z_{k}$, and the series $\sum_{k=1}^{\infty} z_{k} v_{k}$ converges, for this is just the series of $z$.

Suppose now that (iii) holds, and renorm $E$ so that the basis $\left(v_{k}\right)_{k \in \mathbb{N}}$ is monotone. Let $B$ be the unit ball of this norm $\|\cdot\|$. Since $B$ is $\tau_{\mathrm{p}}$-bounded, we only have to prove its pointwise closedness. Let $\left(x_{i}\right) \subset B$ be a net such that for each $k, v_{k}^{\prime}\left(x_{i}\right) \underset{i}{\rightarrow} z_{k}$, and consider any $n \in \mathbb{N}$ and $\varepsilon>0$. Then

$$
\left\|\sum_{k=1}^{n} z_{k} v_{k}\right\| \leq\left\|\sum_{k=1}^{n}\left(z_{k}-v_{k}^{\prime}\left(x_{i}\right)\right) v_{k}\right\|+\left\|\sum_{k=1}^{n} v_{k}^{\prime}\left(x_{i}\right) v_{k}\right\| \leq \varepsilon+1,
$$

where $i$ is large enough. Since $\left(v_{k}\right)$ is boundedly complete, $\sum_{k=1}^{\infty} z_{k} v_{k}$ converges, say to $z \in E$. Now

$$
\|z\|=\lim _{n \rightarrow \infty}\left\|\sum_{k=1}^{n} z_{k} v_{k}\right\| \leq \varepsilon+1
$$

as before, and therefore $z \in B$.

In the remainder of this section we will consider a Banach space of continuous functions $(\mathcal{F}(U),\|\cdot\|)$ satisfying the following two conditions:

(a) the norm $\|\cdot\|$ is finer than the pointwise topology $\tau_{\mathrm{p}}$ on $\mathcal{F}(U)$,

(b) the evaluation map $\delta: U \rightarrow\left(\mathcal{F}(U)^{\prime},\|\cdot\|\right)$ is continuous.

Note that, in this case, the unit ball $B$ of $(\mathcal{F}(U),\|\cdot\|)$ is an equicontinuous $\tau_{\mathrm{p}}$-bounded set. Our purpose is to construct a Banach space containing $\mathcal{F}(U)$ which admits a strong Banach linearization and which is minimal in some sense. By analogy with [7], we define $\mathcal{F}_{\mathrm{pb}}(U)$ as the space of functions on $U$ which are approximable pointwise by bounded nets in $\mathcal{F}(U)$. Each $f \in \mathcal{F}_{\mathrm{pb}}(U)$ is then the pointwise limit of an equicontinuous net of functions on $U$ and is therefore also continuous on $U$.

For $f \in \mathcal{F}_{\mathrm{pb}}(U)$ we define the triple norm $|\|f \mid\|$ as follows. Let

$$
B_{\mathrm{pb}}=\left\{f: U \rightarrow \mathbb{C}: f \text { is a pointwise limit of a net }\left(f_{i}\right) \subset B\right\} .
$$


Thus,

$$
\mathcal{F}_{\mathrm{pb}}(U)=\bigcup_{r>0} r B_{\mathrm{pb}}
$$

and $B_{\mathrm{pb}}$ is a convex, balanced, absorbing subset of $\mathcal{F}_{\mathrm{pb}}(U)$. We let $\||\cdot|||$ be its Minkowski functional, i.e., $|\|f \mid\|$ is the infimum of the constants $c>0$ such that there is a net in $c B$ converging pointwise to $f$ on $U$. Now, the following observations will be useful.

(i) When considering $f \in B_{\mathrm{pb}}$ as the pointwise limit of a net $\left(f_{i}\right)_{i \in I} \subset B$, the same index set $I$ may be used for all $f$. Indeed, if each $f \in B_{\mathrm{pb}}(U)$ is $f=\lim _{i \in I_{f}} f_{i}$, take

$$
I=\prod_{f \in B_{\mathrm{pb}}} I_{f}, \quad \text { ordered by } \quad i \geq j \Leftrightarrow i_{f} \geq j_{f} \text { for all } f \in B_{\mathrm{pb}} .
$$

Now if $f=\lim _{j \in I_{f}} f_{j}$, define $h_{i}=f_{i_{f}}$, and we have $f=\lim _{i \in I} h_{i}$.

(ii) Conditions (a) and (b) above also hold for $\left(\mathcal{F}_{\mathrm{pb}}(U),\||\cdot|\| \mid\right)$ :

For (a), if $f_{n} \rightarrow 0$ in $\|\cdot\| \|$, let $x \in U$ and $\varepsilon>0$. Choose $n_{\varepsilon}$ so that

$$
\left\|f_{n}\right\|<\frac{\varepsilon}{2\left\|\delta_{x}\right\|} \quad \text { for all } n \geq n_{\varepsilon} .
$$

For each $n$, take a net $\left(f_{n, i}\right) \subset \frac{\varepsilon}{2\left\|\delta_{x}\right\|} B$ converging pointwise to $f_{n}$. Then

$$
\begin{aligned}
\left|f_{n}(x)\right| & \leq\left|f_{n}(x)-f_{n, i}(x)\right|+\left|f_{n, i}(x)\right|=\left|f_{n}(x)-f_{n, i}(x)\right|+\left|\delta_{x}\left(f_{n, i}\right)\right| \\
& \leq\left|f_{n}(x)-f_{n, i}(x)\right|+\left\|\delta_{x}\right\| \cdot\left\|f_{n, i}\right\|
\end{aligned}
$$

but the second term is smaller than $\varepsilon / 2$ for $n \geq n_{\varepsilon}$, and the first is also less than $\varepsilon / 2$ for $i \geq i(n, \varepsilon)$. Thus $\delta: U \rightarrow\left(\mathcal{F}_{\mathrm{pb}}(U), \||| \cdot||\right)^{\prime}$ is well-defined.

To see (b), fix $x \in U$ and $\varepsilon>0$. There is a neighborhood $V_{x}$ of $x$ such that $\left\|\delta_{x}-\delta_{y}\right\|<\varepsilon / 3$ in $(\mathcal{F}(U),\|\cdot\|)^{\prime}$ for all $y \in V_{x}$. Now for any $f$ with $\left\||f \||<1\right.$, let $\left(f_{i}\right) \subset B$ converge pointwise to $f$. Then for any $y \in V_{x}$,

$$
\begin{aligned}
|f(x)-f(y)| & \leq\left|f(x)-f_{i}(x)\right|+\left|f_{i}(x)-f_{i}(y)\right|+\left|f_{i}(y)-f(y)\right| \\
& \leq\left|f(x)-f_{i}(x)\right|+\left|\left(\delta_{x}-\delta_{y}\right)\left(f_{i}\right)\right|+\left|f_{i}(y)-f(y)\right| \\
& \leq\left|f(x)-f_{i}(x)\right|+|| \delta_{x}-\delta_{y}||+\left|f_{i}(y)-f(y)\right| .
\end{aligned}
$$

Now, the second term is smaller than $\varepsilon / 3$ for all $y \in V_{x}$, independently of $f$, while the first and last terms can be made smaller than $\varepsilon / 3$ for sufficiently large $i$.

(iii) The inclusion $\left(\mathcal{F}_{\mathrm{pb}}(U),\|\| \cdot \| \mid\right) \rightarrow\left(C(U), \tau_{\mathrm{co}}\right)$ is continuous: for any compact subset $K$ of $U$, and $\varepsilon>0$,

$$
\|f\|_{K}=\sup _{x \in K}\left|\delta_{x}(f)\right| \leq \sup _{x \in K}\left\|\delta_{x}\right\| \cdot\|f\|\left|=C_{K}\right|\|f\| \| .
$$

(iv) $B_{\mathrm{pb}}$ is the closed unit ball of the norm $\||\cdot|\|$. Indeed, since this norm is the Minkowski functional of $B_{\mathrm{pb}}$, one has

$$
\left\{f \in \mathcal{F}_{\mathrm{pb}}(U):\|f\| \mid<1\right\} \subset B_{\mathrm{pb}} \subset\left\{f \in \mathcal{F}_{\mathrm{pb}}(U):\|f\| \mid \leq 1\right\} ;
$$


but $B_{\mathrm{pb}}$ is pointwise closed, and $\left(\mathcal{F}_{\mathrm{pb}}(U),\|\| \cdot \| \mid\right) \rightarrow\left(\mathcal{F}_{\mathrm{pb}}(U), \tau_{\mathrm{p}}\right)$ is continuous, so $B_{\mathrm{pb}}$ is ||$|\cdot|||-$-closed, and is therefore equal to the closed ||$|\cdot|||$-ball.

We also have

Proposition 2.6. $\left(\mathcal{F}_{\mathrm{pb}}(U),\||\cdot|\|\right)$ is a Banach space, and the inclusion mapping $\iota:(\mathcal{F}(U),\|\cdot\|) \hookrightarrow\left(\mathcal{F}_{\mathrm{pb}}(U),\||\cdot|\|\right)$ is continuous.

Proof. First, we check the completeness. So let $\left(f_{n}\right)$ be a $\|||||-$-Cauchy sequence in $\mathcal{F}_{\mathrm{pb}}(U)$. By passing to a subsequence we may suppose that $\left\|\left|f_{n}-f_{n+1} \|\right|<1 / 2^{n}\right.$ for all $n$. Note also that for each $x \in U,\left(f_{n}(x)\right)$ is Cauchy, so the $f_{n}$ 's converge pointwise to a function $f \in \mathcal{F}_{\mathrm{pb}}(U)$. We must show that it converges also in $\||\cdot|\|$.

We define inductively a sequence of nets in $\mathcal{F}(U)$ as follows: for $n=1$ let $\left(f_{1, i}\right)$ be a bounded net converging pointwise to $f_{1}$. Suppose we have defined nets $\left(f_{1, i}\right), \ldots,\left(f_{n, i}\right)$ such that for each $k=2, \ldots, n$ we have $\left(f_{k-1, i}-f_{k, i}\right) \subset$ $\left(1 / 2^{k-1}\right) B$ and $\left(f_{k, i}\right)$ converges pointwise to $f_{k}$. Now since $\left\|f_{n}-f_{n+1}\right\|<<$ $1 / 2^{n}$, there is a net $\left(h_{n, i}\right) \subset\left(1 / 2^{n}\right) B$ converging pointwise to $f_{n}-f_{n+1}$. We define $f_{n+1, i}=f_{n, i}-h_{n, i}$. Thus $f_{n+1, i}$ converges pointwise to $f_{n}-\left(f_{n}-\right.$ $\left.f_{n+1}\right)=f_{n+1}$, and $\left(f_{n, i}-f_{n+1, i}\right) \subset\left(1 / 2^{n}\right) B$. Now, for each fixed $i$, the sequence $\left(f_{n, i}\right)$ is $\|\cdot\|$-Cauchy in $\mathcal{F}(U)$. Indeed, if $m>n$,

$$
\begin{aligned}
\left\|f_{n, i}-f_{m, i}\right\| & \leq\left\|f_{n, i}-f_{n+1, i}\right\|+\left\|f_{n+1, i}-f_{n+2, i}\right\|+\cdots+\left\|f_{m-1, i}-f_{m, i}\right\| \\
& \leq \frac{1}{2^{n}}+\frac{1}{2^{n+1}}+\cdots+\frac{1}{2^{m}} \\
& <\sum_{k=n}^{\infty} \frac{1}{2^{k}}=\frac{1}{2^{n-1}} .
\end{aligned}
$$

Since $\mathcal{F}(U)$ is a Banach space, $\left(f_{n, i}\right)$ converges in the $\|\cdot\|$-norm, as $n \rightarrow \infty$, to a function $f_{i} \in \mathcal{F}(U)$. From this and $\left\|f_{n, i}-f_{m, i}\right\|<1 / 2^{n-1}$ for all $m>n$, we deduce that $\left\|f_{n, i}-f_{i}\right\| \leq 1 / 2^{n-1}$ for all $i$. Note also that the net $\left(f_{i}\right)$ converges pointwise to $f$. Indeed, fix $x \in U$ and $\varepsilon>0$. Then

$$
\begin{aligned}
\left|f(x)-f_{i}(x)\right| & \leq\left|f(x)-f_{n}(x)\right|+\left|f_{n}(x)-f_{n, i}(x)\right|+\left|f_{n, i}(x)-f_{i}(x)\right| \\
& =\left|f(x)-f_{n}(x)\right|+\left|f_{n}(x)-f_{n, i}(x)\right|+\left|\delta_{x}\left(f_{n, i}-f_{i}\right)\right| \\
& \leq\left|f(x)-f_{n}(x)\right|+\left|f_{n}(x)-f_{n, i}(x)\right|+\left\|\delta_{x}\right\| \cdot\left\|f_{n, i}-f_{i}\right\| \\
& \leq\left|f(x)-f_{n}(x)\right|+\left|f_{n}(x)-f_{n, i}(x)\right|+\left\|\delta_{x}\right\| \frac{1}{2^{n-1}} .
\end{aligned}
$$

Choose $n$ so large that the first and last terms are smaller than $\varepsilon / 3$; then the middle term will also be smaller than $\varepsilon / 3$ for $i \geq i(n, x)$.

Thus, for each $n$, we have a net $\left(f_{n, i}-f_{i}\right)$ converging pointwise to $f_{n}-f$, and such that $\left(f_{n, i}-f_{i}\right) \subset\left(1 / 2^{n-1}\right) B$, in other words, $\left\|\left|f_{n}-f\right|\right\| \leq 1 / 2^{n-1}$. Hence $\left(\mathcal{F}_{\mathrm{pb}}(U),\|\| \cdot \|\right)$ is a Banach space.

Finally, it is clear that $\mathcal{F}(U) \subset \mathcal{F}_{\mathrm{pb}}(U)$ and \|\|$f\|\mid \leq\| f \|$ for every $f$ in $\mathcal{F}(U)$. 
Corollary 2.7. The space $\left(\mathcal{F}_{\mathrm{pb}}(U), \||\cdot|||\right)$ always admits a strong $B a$ nach linearization. Moreover, $(\mathcal{F}(U),\|\cdot\|)$ admits a strong Banach linearization if and only if $\mathcal{F}(U)=\mathcal{F}_{\mathrm{pb}}(U)$.

Proof. The unit ball $B_{\mathrm{pb}}$ of $\mathcal{F}_{\mathrm{pb}}(U)$ is $\tau_{\mathrm{p}^{-}}$-compact, since it is the $\tau_{\mathrm{p}^{-}}$ closure of the ball $B$ of $\mathcal{F}(U)$. Since $\mathcal{F}_{\mathrm{pb}}(U)$ satisfies condition (b), a direct application of Theorem 2.2 shows that $\mathcal{F}_{\mathrm{pb}}(U)$ admits a strong Banach linearization.

On the other hand, if $\mathcal{F}(U)$ admits a strong Banach linearization, it has an equivalent $\tau_{\mathrm{p}}$-compact unit ball, so $B_{\mathrm{pb}}=B$ and $\mathcal{F}(U)=\mathcal{F}_{\mathrm{pb}}(U)$. Conversely, if this equality holds, by the Open Mapping Theorem, the norms $\|\cdot\|$ and ||$|\cdot|||$ are equivalent and the result follows.

The minimality of $\mathcal{F}_{\mathrm{pb}}(U)$ has to be understood in the following sense.

Proposition 2.8. Let $\mathcal{G}(U)$ be a Banach space of continuous functions on $U$ containing $\mathcal{F}(U)$ with continuous inclusion $\iota: \mathcal{F}(U) \hookrightarrow \mathcal{G}(U)$. If $\mathcal{G}(U)$ admits a strong Banach linearization, then $\mathcal{G}(U)$ also contains $\mathcal{F}_{\mathrm{pb}}(U)$ with continuous inclusion.

Proof. By Theorem 2.2, we can consider an equivalent norm $\|\cdot\|_{\mathcal{G}}$ on $\mathcal{G}(U)$, whose unit ball is $\tau_{\mathrm{p}}$-compact. By the continuity of $\iota$, there exists $C>0$ such that $\|f\|_{\mathcal{G}} \leq C\|f\|$ for any $f \in \mathcal{F}(U)$. Now, each $f \in \mathcal{F}_{\mathrm{pb}}(U)$ belongs to $\mathcal{G}(U)$, as $f$ is the $\tau_{\mathrm{p}}$-limit of a $\|\cdot\|_{\mathcal{G}^{-}}$-bounded net in $\mathcal{F}(U)$. Finally, it is easy to see that $\|f\|_{\mathcal{G}} \leq C \mid\|f\| \|$ for every $f \in \mathcal{F}_{\mathrm{pb}}(U)$.

In this sense, for Banach spaces of continuous functions satisfying conditions (a) and (b), to admit a strong Banach linearization is equivalent to being saturated with respect to the pointwise limits of bounded nets. This is the case for the spaces of $k$-homogeneous polynomials on a Banach space, the space of Lipschitz functions on a metric space, and the space of bounded holomorphic functions on the unit ball of a Banach space (endowed with their natural norms). Nevertheless, if we consider, as in [7], a dual Banach space $Z$ and the uniform algebra $\mathcal{A}(B)$ generated by the weak-star continuous linear functionals on the closed unit ball $B$ of $Z$, this is a non-saturated subalgebra of $\mathcal{H}^{\infty}(B)$. In this case, $\mathcal{A}_{\mathrm{pb}}(B)=\mathcal{H}^{\infty}(B)$ only under certain assumptions on $Z$ (for example, when $Z$ has the metric approximation property; see [7, Theorem 4.4]).

Finally, we mention the case of Banach spaces $E=\mathcal{F}(\mathbb{N})$. Those which admit a strong Banach linearization are those saturated with respect to the pointwise limits of bounded nets (for example, $\ell^{\infty}$ ). As mentioned before, $c_{0}$ does not admit a strong Banach linearization and it is non-saturated.

3. Compactness properties. We now focus on compactness properties of mappings and of their linearizations. In this section, $\mathcal{F}(U)$ denotes 
a locally convex space of continuous functions on $U$, which need not be a Banach space. We begin with the definition of several kinds of compactness.

Definition. Let $U$ be a topological space, and $E$ and $F$ locally convex spaces. We say that a mapping $f: U \rightarrow F$ is compact if for every $x \in U$ there is a neighborhood $U_{x}$ of $x$ such that $f\left(U_{x}\right)$ is precompact. Similarly, a linear operator $L: E \rightarrow F$ is compact if there is a neighborhood $V$ of 0 such that $L(V)$ is precompact.

We say that a mapping $f: U \rightarrow F$ is $\mathcal{F}$-boundedly compact if for every $\mathcal{F}$-bounding subset $A \subset U, f(A)$ is precompact. Similarly, a linear operator $L: E \rightarrow F$ will be called boundedly compact if for all bounded subsets $B \subset E, L(B)$ is precompact.

Clearly, compact linear operators are boundedly compact, and the two notions coincide when $E$ is a Banach space.

We are going to see that $\mathcal{F}$-bounded compactness of $f$ is equivalent to bounded compactness of $L_{f}$ in quite general situations. We suppose that $\mathcal{F}(U)$ is barreled and has the topology of uniform convergence on $\mathcal{F}$ bounding subsets of $U$ in order to apply Fact 0.3.

For a linear operator $L: E \rightarrow F$ we denote its transpose by $L^{t}: F^{\prime} \rightarrow$ $E^{\prime}$. In a similar way, for $f \in w \mathcal{F}(U, F)$, we denote by $f^{t}: F^{\prime} \rightarrow \mathcal{F}(U)$ the transpose of $f$ defined by $f^{t}(\varphi)=\varphi \circ f$. The following result relates bounded compactness of a mapping, of its linearization, and of its transpose. Here we denote by $\beta$ and $\tau_{\text {co }}$ the strong and the compact-open topologies, respectively.

TheOREM 3.1. Suppose $\mathcal{F}(U)$ is barreled and has the topology of uniform convergence on $\mathcal{F}$-bounding subsets of $U$. Let $F$ be a locally convex space and $f \in w \mathcal{F}(U, F)$. The following conditions are equivalent.

(i) The mapping $f: U \rightarrow F$ is $\mathcal{F}$-boundedly compact.

(ii) The linearization $L_{f} \in \mathcal{L}\left(\mathcal{F}_{*}(U), F\right)$ is boundedly compact.

If, in addition, $F$ is a complete barreled space, these conditions are also equivalent to the following:

(iii) The transpose operator $\left(L_{f}\right)^{t}:\left(F^{\prime}, \beta\right) \rightarrow\left(\mathcal{F}_{*}(U)^{\prime}, \beta\right)$ is boundedly compact.

(iv) The transpose map $f^{t}:\left(F^{\prime}, \beta\right) \rightarrow \mathcal{F}(U)$ is boundedly compact.

(v) The transpose map $f^{t}:\left(F^{\prime}, \tau_{\mathrm{co}}\right) \rightarrow \mathcal{F}(U)$ is continuous.

Proof. (i) $\Rightarrow$ (ii): Let $f$ be $\mathcal{F}$-boundedly compact and $B \subset \mathcal{F}_{*}(U)$ bounded. By Fact $0.3, U$ has the $B B \mathcal{F}$-property, that is, there exist an $\mathcal{F}$-bounding subset $A \subset U$ and an $r>0$ such that $B \subset r \operatorname{coe}(e(A))$. Then

$$
L_{f}(B) \subset L_{f}(r \operatorname{coe}(e(A)))=r \operatorname{coe}\left(L_{f}(e(A))\right)=r \operatorname{coe}(f(A)),
$$

which is precompact, since $f(A)$ is. Thus $L_{f}$ is boundedly compact. 
(ii) $\Rightarrow\left(\right.$ i): Suppose $L_{f}$ is boundedly compact and $A \subset U \mathcal{F}$-bounding. Since for any $L_{g} \in \mathcal{F}_{*}(U)^{\prime}, L_{g}(e(A))=g(A)$ is bounded, $e(A)$ is $\mathcal{F}$-bounded in $\mathcal{F}_{*}(U)$. Thus $f(A)=L_{f}(e(A))$ is precompact, and $f$ is boundedly compact.

(ii) $\Leftrightarrow$ (iii): If $F$ is barreled, it follows from Proposition 2.1 in [8] that $L_{f}$ is boundedly compact if, and only if, $\left(L_{f}\right)^{t}$ is boundedly compact. Note that in [8] boundedly compact operators are called bpc-operators.

(iii) $\Leftrightarrow\left(\right.$ iv): Note that for every $\gamma \in F^{\prime}$ we have $\gamma \circ L_{f}=L_{\gamma \circ f}$. Since $\mathcal{F}(U)$ is isomorphic to $\left(\mathcal{F}_{*}(U)^{\prime}, \beta\right)$ by Fact 0.3 , we can identify $f^{t}$ with $\left(L_{f}\right)^{t}$, and the result follows.

(i) $\Rightarrow(\mathrm{v})$ : Suppose $f$ is $\mathcal{F}$-boundedly compact and let $B$ be an $\mathcal{F}$-bounding subset of $U$. Since $f(B)$ is precompact, its closure $K$ is a compact subset of the complete space $F$ for which

$$
\sup _{x \in B}\left\{\left|f^{t}(\varphi)(x)\right|\right\}=\sup _{x \in B}\{|(\varphi \circ f)(x)|\} \leq|\varphi|_{K}:=\sup _{x \in K}\{|\varphi(x)|\} \text { for all } \varphi \in F^{\prime} \text {. }
$$

This proves the continuity of $f^{t}$.

$(\mathrm{v}) \Rightarrow(\mathrm{i})$ : If $f^{t}$ is $\tau_{\text {co }}$-continuous, then for any $\mathcal{F}$-bounding subset $B$ of $U$ there exist a compact set $K \subset F$ and $C>0$ so that

$$
\sup _{x \in B}\{|(\varphi \circ f)(x)|\} \leq C|\varphi|_{K} \quad \text { for all } \varphi \in F^{\prime} .
$$

This implies that $f(B) \subset C \operatorname{coe}(K)$ and $f$ is $\mathcal{F}$-boundedly compact

The above result applies, for example, to the Fréchet space $\mathcal{F}(U)=$ $\mathcal{H}_{\mathrm{b}}(U)$, the space of holomorphic functions of bounded type on a balanced open subset $U$ of a Banach space; to $\mathcal{F}(U)=\mathcal{P}\left({ }^{k} E\right)$, the space of continuous $k$-homogeneous polynomials on a Banach space $E$; and to $\mathcal{F}(U)=\mathcal{H}^{\infty}(U)$, the space of bounded holomorphic functions on an open subset of a Banach space. (See Examples 1, 3 and 4 in [6].)

As an application, we have the following characterization of $C_{*}(K)$.

Corollary 3.2. $C_{*}(K)$ is the space of regular Borel measures on $K$ with the topology of uniform convergence over compact subsets of $C(K)$.

Proof. Consider $\mathcal{F}(K)=C(K)$, and take $F=C_{*}(K)$. It is clear that $e: K \rightarrow C_{*}(K)$ is $\mathcal{F}$-boundedly compact. By Theorem 3.1, the linearization $L_{e}=\mathrm{id}: C_{*}(K) \rightarrow C_{*}(K)$ is boundedly compact, and hence $C_{*}(K)$ is semi-Montel, and thus semireflexive. Hence, algebraically $C_{*}(K)=C(K)^{\prime}=$ $\mathcal{M}(K)$, the space of regular Borel measures on $K . C_{*}(K)$ is then the space of regular Borel measures on $K$ with the $\alpha$-topology: uniform convergence over compact subsets of $C(K)$.

A result analogous to Theorem 3.1, but for true compactness, seems to require more of the functions in $\mathcal{F}(U)$, and certainly does not hold for the space of all continuous functions: 
Example 3. Consider $\mathcal{F}(K)=C(K)$, and $F=C_{*}(K)$ as in Corollary 3.2. It is clear that $e: K \rightarrow C_{*}(K)$ is compact. Nevertheless, the linearization $L_{e}=\mathrm{id}: C_{*}(K) \rightarrow C_{*}(K)$ is compact only when $\operatorname{dim} C_{*}(K)<\infty$, or equivalently, when $K$ is finite.

In the context of holomorphic functions, we have the following theorem.

Theorem 3.3. Suppose that $E$ and $F$ are locally convex spaces, $U$ is a connected open subset of $E$, and $\mathcal{F}(U)$ is a linear space of holomorphic functions on $U$. For $f \in w \mathcal{F}(U, F)$, the following are equivalent.

(i) The mapping $f: U \rightarrow F$ is compact.

(ii) For every $x \in U$ there is a neighborhood $W$ of $x$ such that the linearization $L_{\left.f\right|_{W}} \in \mathcal{L}\left(\mathcal{F}_{*}(W), F\right)$ is compact, where $\mathcal{F}(W)=\left\{\left.g\right|_{W}\right.$ : $g \in \mathcal{F}(U)\}$.

Proof. (i) $\Rightarrow$ (ii): Let $W$ be a neighborhood of $x$ such that $f(W)$ is precompact. Note that since $U$ is connected, we can identify $\mathcal{F}(U)=\mathcal{F}(W)$ and therefore $\left(\mathcal{F}_{*}(W), e\right)$ can be seen as a linearization of $\mathcal{F}(U)$. Denote by $\Delta$ the closed unit disk in $\mathbb{C}$, and define

$$
D=\{g \in \mathcal{F}(U): g(W) \subset \Delta\} .
$$

We begin by noting that $D$ is an equicontinuous $\tau_{\mathrm{p}}$-compact disk of $\mathcal{F}(W)$ ([9, Lemma 3.25]). Thus, the set $V=D^{\circ}$ is a neighborhood of zero in the $\alpha$-topology, by [6]. Note that $e(W)^{\circ}=D$, so $V=e(W)^{\circ \circ}=\operatorname{coe}(e(W))$ by the bipolar theorem. Denote by $L_{f}$ the linearization of $f$ by $\mathcal{F}_{*}(W)$. Now

$$
L_{f}(V)=L_{f}(\operatorname{coe}(e(W)))=\operatorname{coe}\left(L_{f}(e(W))\right)=\operatorname{coe}(f(W))
$$

is precompact (see, e.g., [14]). Hence $L_{f}$ is compact.

(ii) $\Rightarrow\left(\right.$ i): For each $x \in U$ let $W$ be as in (ii), and $L_{f}$ the compact linearization of $f$ by $\mathcal{F}_{*}(W)$. Suppose $V$ is a neighborhood of 0 such that $L_{f}(V)$ is precompact, and take $W_{x}=e^{-1}\left(e_{x}+V\right)$. Then $W_{x}$ is a neighborhood of $x$, and

$$
f\left(W_{x}\right)=\left(L_{f} \circ e\right)\left(e^{-1}\left(e_{x}+V\right)\right) \subset L_{f}\left(e_{x}+V\right)=f(x)+L_{f}(V)
$$

is precompact.

For the space $\mathcal{P}\left({ }^{k} E, F\right)$ of continuous $k$-homogeneous polynomials between Banach spaces and for others with Banach linearizations, such as $\mathcal{H}^{\infty}(U)$, a bit more can be said:

Corollary 3.4. Suppose $U$ is a connected open subset of a Banach space, $\mathcal{F}(U)$ is a linear space of holomorphic functions on $U$, and $\mathcal{F}_{*}(U)$ is a Fréchet space. Let $F$ be a locally convex space and $f \in w \mathcal{F}(U, F)$. The following are equivalent.

(i) The mapping $f: U \rightarrow F$ is compact.

(ii) The linearization $L_{f} \in \mathcal{L}\left(\mathcal{F}_{*}(U), F\right)$ is compact. 
Proof. Simply apply Theorem 3.3 and Proposition 1.1.

It is easily seen that the above corollary is false without the Fréchet condition on $\mathcal{F}_{*}(U)$ :

EXAMPLE 4. Take as $U$ any locally compact space and $\mathcal{F}(U)$ an infinitedimensional space of holomorphic functions on $U$ (for example, $\mathcal{F}(U)=$ $\mathcal{H}(\mathbb{C})$, the entire functions on the complex plane). Choose $F$ to be $\mathcal{F}_{*}(U)$. Then $e: U \rightarrow \mathcal{F}_{*}(U)$ is compact, but its linearization is the identity $I$ : $\mathcal{F}_{*}(U) \rightarrow \mathcal{F}_{*}(U)$, which cannot be compact.

REMARK. Note that in the previous corollary, analyticity of the functions in $\mathcal{F}(U)$ was used only to prove that the set $D=\{g \in \mathcal{F}(U): g(W) \subset \Delta\}$ is equicontinuous. There are other spaces $\mathcal{F}(U)$ where this is so, and therefore the theorem is also valid for them. A case in point is the space $\operatorname{Lip}(U)$ of Lipschitz functions on a metric space $U$, endowed with its natural norm.

We now combine our results with factorization theorems for linear operators to obtain the following result, which should be compared with [22]. Here, we say that a mapping $f: U \rightarrow F$ is weakly compact if for every $x \in U$ there is a neighborhood $U_{x}$ of $x$ such that $f\left(U_{x}\right)$ is weakly precompact. Similarly, a linear operator $L: E \rightarrow F$ is weakly compact if there is a neighborhood $V$ of 0 such that $L(V)$ is weakly precompact.

Corollary 3.5. Suppose $U$ is a connected open subset of a Banach space, $\mathcal{F}(U)$ is a linear space of holomorphic functions on $U$, and $\mathcal{F}_{*}(U)$ is a Fréchet space. Let $F$ be a Fréchet space and $f \in w \mathcal{F}(U, F)$. The following are equivalent.

(i) The mapping $f: U \rightarrow F$ is weakly compact.

(ii) The linearization $L_{f} \in \mathcal{L}\left(\mathcal{F}_{*}(U), F\right)$ is weakly compact.

(iii) The mapping $f: U \rightarrow F$ factors through a reflexive Banach space. That is, there exist a reflexive Banach space $Z$, a mapping $g \in$ $w \mathcal{F}(U, Z)$, and a continuous linear operator $T: Z \rightarrow F$ such that

$$
f=T \circ g .
$$

Proof. The equivalence between (i) and (ii) holds for every locally convex space $F$, and follows from Corollary 3.4 applied to the space ( $F$, weak). To prove (ii) $\Rightarrow($ iii), we apply the Davis-Figiel-Johnson-Pełczyński factorization theorem for linear operators (see e.g. [14, Theorem 17.2.9]). It yields a reflexive Banach space $Z$ and continuous linear operators $S: \mathcal{F}_{*}(U) \rightarrow Z$ and $T: Z \rightarrow F$ such that $L_{f}=T \circ S$. Then $f$ factors through $Z$ since $f=T \circ(S \circ e)$ and $S \circ e \in w \mathcal{F}(U, Z)$. Finally, (iii) $\Rightarrow(\mathrm{i})$ is clear.

Acknowledgements. It is a pleasure to thank José Bonet and Manuel Maestre for their valuable comments about this paper. The third author 
would also like to thank the Departamento de Análisis Matemático of the Universidad Complutense de Madrid for its recurrent hospitality.

The first two authors have been partially supported by MEC grant MTM 2006-03531 and by CAM-910626, and the third author by an 'Estancia de Profesor Extranjero' grant from Grupo Santander/UCM, 2006.

\section{References}

[1] R. M. Aron and M. Schottenloher, Compact holomorphic mappings on Banach spaces and the approximation property, J. Funct. Anal. 21 (1976), 7-30.

[2] K. Bierstedt and J. Bonet, Biduality in Fréchet and (LB)-spaces, in: Progress in Functional Analysis, K. Bierstedt et al. (eds.), North-Holland, Amsterdam, 1992, 113-135.

[3] C. Boyd, Montel and reflexive preduals of spaces of holomorphic functions on Fréchet spaces, Studia Math. 107 (1993), 305-315.

[4] —, Distinguished preduals of spaces of holomorphic functions, Rev. Mat. Complut. 6 (1993), 221-231.

[5] E. Çalişkan, The bounded approximation property for the predual of the space of bounded holomorphic mappings, Studia Math. 177 (2006), 225-233.

[6] D. Carando and I. Zalduendo, Linearization of functions, Math. Ann. 328 (2004), 683-700.

[7] T. K. Carne, R. Cole and T. W. Gamelin, A uniform algebra of analytic functions on a Banach space, Trans. Amer. Math. Soc. 314 (1989), 638-659.

[8] S. Dierolf and P. Domański, Factorization of Montel operators, Studia Math. 107 (1993), 15-32.

[9] S. Dineen, Complex Analysis in Infinite Dimensional Spaces, Springer Monogr. Math., Springer, London, 1999.

[10] M. Fabian, P. Habala, P. Hájek, V. Montesinos, J. Pelant and V. Zizler, Functional Analysis and Infinite-Dimensional Geometry, CMS Books in Mathematics/Ouvrages de Mathématiques de la SMC 8, Springer, New York, 2001.

[11] P. Galindo, D. García and M. Maestre, Holomorphic mappings of bounded type, J. Math. Anal. Appl. 166 (1992), 236-246.

[12] D. García and J. Mujica, Quasi-normable preduals of spaces of holomorphic functions, ibid. 208 (1997), 171-180.

[13] A. Grothendieck, Une caractérisation vectorielle-métrique des espaces $L^{1}$, Canad. J. Math. 7 (1955), 552-561.

[14] H. Jarchow, Locally Convex Spaces, Teubner, Stuttgart, 1981.

[15] P. Mazet, Analytic Sets in Locally Convex Spaces, North-Holland Math. Stud. 89, North-Holland, Amsterdam, 1984.

[16] J. Mujica, Linearization of bounded holomorphic mappings on Banach spaces, Trans. Amer. Math. Soc. 324 (1991), 867-887.

[17] —, Linearization of holomorphic mappings of bounded type, in: Progress in Functional Analysis, North-Holland, Amsterdam, 1992, 149-162.

[18] J. Mujica and L. Nachbin, Linearization of holomorphic mappings on locally convex spaces, J. Math. Pures Appl. 71 (1992), 543-560.

[19] K. Ng, On a theorem of Dixmier, Math. Scand. 29 (1971), 279-280. 
[20] A. Pełczyński, On weakly compact polynomial operators on B-spaces with DunfordPettis property, Bull. Acad. Polon. Sci. Sér. Sci. Math. Astronom. Phys. 11 (1963), 371-378.

[21] R. Ryan, Applications of topological tensor products to infinite dimensional holomorphy, Ph.D. thesis, Univ. College, Dublin, 1980.

[22] - Weakly compact holomorphic mappings on Banach spaces, Pacific J. Math. 131 (1988), 179-190.

[23] N. Weaver, Lipschitz Algebras, World Sci., Singapore, 1999.

Departamento de Análisis Matemático

Facultad de Ciencias Matemáticas

Universidad Complutense de Madrid

28040 Madrid, Spain

E-mail: jaramil@mat.ucm.es

angelin@mat.ucm.es
Departamento de Matemática Universidad Torcuato Di Tella

Miñones 2177 (C1428ATG)

Buenos Aires, Argentina

E-mail: nacho@utdt.edu 